\title{
Riqueza de espécies de aves no Parque Estadual do Pico do Jabre, Paraíba
}

\author{
Erich de Freitas Mariano ${ }^{a *}$, Luana Régia Alves Martins ${ }^{\mathrm{b}}$ \\ a Unidade Acadêmica de Ciências Biológicas, Universidade Federal de Campina Grande, Patos, 58708-110, Brasil. *efmariano.ufcg@gmail.com \\ ${ }^{b}$ Unidade Acadêmica de Ciências Biológicas, Universidade Federal de Campina Grande, Patos, 58708-010, Brasil.
}

Recebido: 14 junho 2017 / Aceito: 31 julho 2017 / Publicado online: 27 setembro 2017

\begin{abstract}
Resumo
O presente estudo teve como objetivo fazer um levantamento das espécies de aves do Parque Estadual do Pico do Jabre, Paraíba, listando as espécies em relação as suas categorias tróficas e dependência de ambientes florestais. Foram montadas 240 listas de Mackinnon em 12 dias de amostragem. A família que apresentou maior riqueza foi Tyrannidae, com 20 espécies, seguida de Thamnophilidae (7), Trochilidae (6) e Emberizidae (6). Aproximadamente 20\% das espécies que ocorrem na área se mostraram dependentes de ambientes florestais, 38,7\% semi-dependentes e 41,4\% independentes. A baixa participação de espécies dependentes de ambientes florestais na comunidade pode estar associada a pressão sofrida no entorno do parque. A manutenção destas áreas se mostra importante devido ao intercâmbio de espécies com as áreas secas adjacentes, servindo de refúgios para estas espécies nos períodos de seca. A presença da Penelope jacucaca (jacu-caca), que está inserida no livro vermelho de espécies da fauna brasileira ameaçada de extinção, é um dos indicativos que apesar do entorno do parque sofrer com a presença do homem, a área florestal do parque encontra-se em bom estado de preservação.
\end{abstract}

Palavras-chave: Caatinga, Floresta Estacional Semidecidual Montana, Unidades de conservação, Ornitofauna

\section{Bird species richness at Parque Estadual do Pico do Jabre, Paraíba}

\begin{abstract}
This study aims to make an inventory of bird species from Parque Estadual do Pico do Jabre, Paraíba, listing trophic guilds and forest habitat dependencies. In 12 days of survey, 240 Mackinnon lists was builted. Tyrannidae was the richest bird family, with 20 species, followed by Thamnophilidae (7), Trochilidae (6) and Emberezidae (6). Almost 20\% of registered bird species are forest habitat dependent, $38.7 \%$ are semi-dependent and $41.4 \%$ are independent. The low occurrence of forest dependent species should be associated of antropogenic pressions around the park. The maintenance of these areas is important due to the exchange of species with the adjacent dry areas, serving as refuges for these species during the dry periods. The presence of Penelope jacucaca (jacu-caca), which is inserted in the red book of Brazilian threatened species of fauna, is an indicator that despite the surroundings of the park suffer with the presence of man, the forest area of the park is in good condition preservation.
\end{abstract}

Keywords: Caatinga, Montane Seasonal Semideciduous Forest, Conservation Units, Bird fauna.

\section{Introdução}

A floresta estacional semidecidual Montana, os chamados "brejos de altitude", ocorrem na região nordeste do Brasil nos topos de serras dentro dos domínios da Caatinga, em uma região semi-árida onde a precipitação varia de $240-900 \mathrm{~mm}$ (IBGE, 1985). As assim chamadas "ilhas de florestas" associam-se a ocorrência de planaltos e chapadas entre 500$1300 \mathrm{~m}$ altitude (e.g., Borborema, chapada do Araripe, Chapada de Ibiapaba), onde as chuvas orográficas garantem níveis de precipitação superiores a $1200 \mathrm{~mm} /$ ano (AndradeLima, 1982; Tabarelli e Santos, 2004).

A vegetação do Pico do Jabre é classificada como Floresta Estacional Semidecidual Montana (IBGE, 1985), e de acordo com Oliveira-Filho e Fontes (2000), ocorre dentro do domínio da Mata Atlântica, com elementos de mata úmida e da
Caatinga (Agra et al., 2004). Entretanto, Rodal et al. (2008) demonstraram que florestas estacionais no nordeste brasileiro, incluindo florestas de altitude, compõem dois grupos florísticos, um associado com a floresta Atlântica costeira e outro com o domínio das caatingas, e fornece a hipótese de que o Planalto da Borborema, uma série de maciços direcionados na posição norte-sul, funciona como uma barreira geográfica que separa as duas floras.

As florestas localizadas no planalto apresentariam, portanto, uma diferenciação: mais ao leste teriam uma origem as mais relacionadas com a floresta Atlântica costeira e, mais ao oeste, as mais relacionadas com vegetações do domínio das caatingas. Essa proposta é suportada através da análise de distribuição de aves residentes e dependentes de ambientes florestais, as quais agrupam as formações a oeste do Planalto da Borborema com as formações de mata seca. 
Tabarelli e Santos (2004) classificaram a vegetação do Pico do Jabre como floresta Ombrófila Montana, e com a menor cobertura vegetal $\left(5,52 \mathrm{~km}^{2}\right)$, durante 15 áreas em Pernambuco e Paraíba, abaixo de 1000 ha., sendo considerada uma área de altíssima prioridade para a conservação.

A avifauna da caatinga, possui 510 espécies de aves que habitam a região (Silva et al., 2003), um número bem superior ao que era esperado. Contudo, para chegar a esse número, foram incluídas as espécies também registradas nos enclaves úmidos. Sua inclusão foi justificada apela inexistência de suposta razão biogeográfica para tratar tais formações como não pertencentes a caatinga (Ab'saber, 1970, 1977), e também devido a sua importância na manutenção de processos ecológicos regionais, como sítios de "refúgio" durante o período de estiagem (Silva et al., 2003).

$\mathrm{O}$ presente estudo teve como objetivo fazer um levantamento das espécies de aves do Parque Estadual do Pico do Jabre, Paraíba, listando as espécies quanto as suas categorias tróficas e dependência de ambientes florestais.

\section{Material e Métodos}

O Parque Estadual do Pico do Jabre situa-se entre os municípios de Maturéia e Mãe D'água entre os paralelos $06^{\circ}$ $02^{\prime} 12^{\prime \prime}$ e $08^{\circ} 19^{\prime} 18^{\prime \prime}$ ' sul e entre os meridianos de $34^{\circ} 45^{\prime} 12^{\prime \prime}$ ' e $38^{\circ} 45^{\prime} 45^{\prime}$ ' Oeste. Sendo o ponto mais elevado da Paraíba $(1.197 \mathrm{~m})$ e do Nordeste Setentrional. A Serra de Teixeira, onde se encontra o Pico do Jabre, está no setor do Planalto Central da Borborema, no trecho compreendido pelo povoado de Cacimbas. Seu formato é alongado, com direção WSWENE, com cerca de $100 \mathrm{Km}$ de extensão e $10 \mathrm{Km}$ de largura.

Além das formações florestais com árvores atingindo até $10 \mathrm{~m}$ de altura, são encontradas plantações, zonas desmatadas, ou áreas abertas e manchas de áreas arbóreas, que seriam áreas com cobertura dominante de árvores, porém diferindo dos ambientes florestais pela presença de uma maior proporção de arbustos e ervas no sub-bosque.

As atividades de amostragem tiveram 12 dias de duração e contou com a aplicação de listas de McKinnon (Bibby et al., 1998). As listas de Mackinnon (Mackinnon e Philips, 1993) compreenderam listas de 10 espécies, as quais foram utilizadas como unidades amostrais. Por dia de amostragem foram construídas 20 listas, totalizando ao final 240 listas e 2400 contatos por localidade. Nessas amostragens, foi utilizado microfone unidirecional e um gravador digital Marantz 670 para registros sonoros das espécies, bem como para utilização da técnica de playback para atrair espécies e facilitar sua identificação.

Curvas acumulativas e de rarefação foram calculadas a partir de dados de presença e ausência da avifauna nas áreas estudadas obtidos durante as amostragens em campo. Uma estimativa de riqueza de espécies foi realizada com os estimadores Chao 2 e Jack 1, por terem apresentados melhor performance em testes de estimadores realizados por Araujo et al. (2012). As análises referentes à curva de rarefação e estimativa de riqueza de espécies foram realizadas utilizando o software EstimateS 7.5 (Colwell, 2005).

Estatísticas descritivas foram utilizadas para comparar a avifauna e a distribuição da porcentagem das categorias de uso de habitat nos ambientes registrados.

\section{Resultados e Discussão}

Foram registradas 111 espécies de aves distribuídas em 35 famílias. As famílias que apresentaram maior riqueza foi Trochilidae com 6 espécies, seguida de Tinamidae, Columbidae e Cuculidae, todas estas com 4 espécies, dentre os Não-Passeriformes; Entre os Passeriformes, as famílias Tyrannidae (20), Thamnophilidae (7) e Emberizidae (6) foram as mais ricas (Tabela 1). As espécies mais frequentes nas amostragens foram Cantorchilus longirostris (Vieillot, 1819) (57,5\%), Tyrannus melancholicus Vieillot, 1819 (52,9\%), Taraba major (Vieillot, 1816) (51,7\%), e Sporophila nigricollis (Vieillot, 1823) (41,3\%).

Dentre as espécies registradas, apenas $P$. jacucaca Spix, 1925 está inserida no livro vermelho de espécies da fauna brasileira ameaçada, sendo considerada vulnerável de acordo com Silveira e Straube (2008). P. jacucaca tem uma fundamental importância para a manutenção de ambientes florestais, por ser uma espécie frugívora e, ao sair na busca de recursos alimentares, realiza a dispersão de sementes.

A presença de espécies como Anopetia gounellei (Boucard, 1891), Conirostrum speciosum (Temminck, 1824), Formicivora melanogaster (Pelzeln, 1868), Gyalophylax hellmayri (Reiser, 1905), Sakesphorus cristatus (Wied, 1831) e Thamnophilus pelzelni (Hellmayr, 1924), são relevantes, pois fundamentam as relações do Pico do Jabre com as formações florestais de mata seca.

Não foram observadas muitas espécies migratórias. Apenas Elaenia chilensis (Hellmayr, 1927), Sporophila lineola (Linnaeus, 1758) e Vireo Chivi (Vieillot, 1817) apresentam deslocamentos sazonais ao longo da América do Sul. Outras espécies como Coccyzus melacoryphus (Vieillot, 1817), Sporophila albogularis (Spix, 1825) e os Columbidae apresentam deslocamentos locais, acompanhando o regime de chuvas.

Algumas espécies foram inventariadas através de encontros visuais ou sonoros ocasionais e a uma curva de acumulação de espécies foi obtida analisando-se apenas a riqueza das espécies registradas pelas listas de McKinnon. Quando se analisou a riqueza estimada de espécies através da curva de rarefação verificamos que a curva obtida através dos dados das listas de MacKinnon não tende a estabilização, sugerindo que uma parte das espécies que ocorrerem na área ainda não foram registradas e os valores das estimativas de Chao 2 e Jack 1 foram de 159 e 134 espécies, respectivamente (Figura 1).

Para nos aproximarmos da riqueza real de uma determinada área percebemos a necessidade de aumentarmos o esforço amostral, conduzido por longos períodos (Santos et al. 2010) e que, com a continuidade dos levantamentos na área de estudo, o número de espécies registradas tende a aumentar, em função, principalmente, daqueles elementos que apresentam baixa densidade populacional, cujo registro é dificultado em curtos períodos de levantamento. 
Tabela 1. Lista de espécies de aves classificadas por ordem sistemática registradas no Parque Estadual do Pico do Jabre, Paraíba e suas respectivas frequências de ocorrência (FO\%) nos diferentes ambientes, categorias tróficas (CT) e uso de habitat (UH).

\begin{tabular}{|c|c|c|c|c|c|c|c|c|c|c|c|}
\hline \multirow[b]{2}{*}{ Espécie } & \multicolumn{3}{|c|}{ FO\% da Área } & \multirow[b]{2}{*}{$\mathrm{CT}$} & \multirow[b]{2}{*}{$\mathrm{UH}$} & \multirow[b]{2}{*}{ Espécie } & \multicolumn{3}{|c|}{ FO\% da Área } & \multirow[b]{2}{*}{$\mathrm{CT}$} & \multirow[b]{2}{*}{$\mathrm{UH}$} \\
\hline & Arbórea & $\begin{array}{c}\text { Área } \\
\text { aberta }\end{array}$ & Floresta & & & & Arbórea & $\begin{array}{c}\text { Área } \\
\text { aberta }\end{array}$ & Floresta & & \\
\hline Tinamidae & & & & & & Strigidae & & & & & \\
\hline Crypturellus noctivagus & 0,4 & - & 0,8 & FRU & DEP & Pulsatrix perspicillata & 0,4 & - & - & CAR & DEP \\
\hline Crypturellus parvirostris & 0,8 & 0,4 & 1,3 & FRU & IND & Glaucidium brasilianum & - & - & 0,8 & CAR & SDE \\
\hline Crypturellus tataupa & 5,4 & 3,3 & 7,9 & FRU & DEP & Athene cunicularia & - & 1,3 & - & CAR & IND \\
\hline Nothura boraquira & - & 0,4 & - & FRU & SDE & Nyctibiidae & & & & & \\
\hline Cracidae & & & & & & Nyctibius griseus & 0,8 & 0,4 & - & INS & SDE \\
\hline Penelope superciliaris & 0,8 & - & 1,3 & FRU & DEP & Caprimulgidae & & & & & \\
\hline P. jacucaca & - & - & 0,4 & FRU & DEP & Antrostomus rufus & 0,4 & - & - & INS & SDE \\
\hline Ardeidae & & & & & & Hydropsalis albicollis & 0,4 & - & - & INS & SDE \\
\hline Butorides striata & - & 2,5 & 0,8 & AQUA & IND & Apodidae & & & & & \\
\hline Bubulcus íbis & - & 2,5 & 1,7 & IND & IND & Tachornis squamata & - & - & 1,3 & INS & IND \\
\hline Ardea alba & - & 1,3 & - & AQUA & IND & Trochilidae & & & & & \\
\hline Egretta thula & - & 0,8 & - & AQUA & IND & Anopetia gounellei & 0,4 & - & - & NEC & DEP \\
\hline Cathartidae & & & & & & Phaethornis pretrei & 2,5 & - & 5,8 & NEC & SDE \\
\hline Cathartes aura & 1,3 & 1,3 & 3,3 & DET & IND & Anthracothorax nigricollis & - & - & 0,4 & NEC & SDE \\
\hline Coragyps atratus & - & - & 1,7 & DET & IND & Chrysolampis mosquitus & 0,4 & - & 0,8 & NEC & IND \\
\hline Accipitridae & & & & & & Chlorostilbon lucidus & 3,8 & 0,4 & 5,4 & NEC & SDE \\
\hline Rupornis magnirostris & 6,7 & 1,7 & 11,7 & CAR & IND & Amazilia leucogaster & 1,3 & 0,4 & 7,1 & NEC & DEP \\
\hline Geranoaetus melanoleucus & - & - & 0,4 & CAR & IND & Bucconidae & & & & & \\
\hline Falconidae & & & & & & Nystalus maculatus & - & - & 0,4 & INS & SDE \\
\hline Caracara plancus & - & 0,4 & - & CAR & IND & Picidae & & & & & \\
\hline Milvago chimachima & - & - & 1,3 & CAR & IND & Veniliornis passerinus & 2,1 & - & 2,9 & INS & SDE \\
\hline Falco femoralis & - & - & 0,4 & CAR & IND & Picumnus fulvescens & 2,1 & - & 2,1 & INS & SDE \\
\hline Charadriidae & & & & & & Thamnophilidae & & & & & \\
\hline Vanellus chilensis & 1,7 & 2,1 & 0,4 & AQUA & IND & Myrmorchilus strigilatus & 12,9 & 2,1 & 14,2 & INS & SDE \\
\hline Jacanidae & & & & & & Formicivora melanogaster & 3,8 & - & 3,3 & INS & SDE \\
\hline Jacana jacana & - & 0,4 & - & AQUA & IND & Herpsilochmus atricapillus & 9,6 & - & 14,6 & INS & DEP \\
\hline Columbidae & & & & & & Sakesphorus cristatus & 2,9 & - & 2,5 & INS & SDE \\
\hline Columbina talpacoti & 10,0 & 6,3 & 9,2 & GRA & IND & Thamnophilus pelzelni & 2,9 & - & 9,2 & INS & SDE \\
\hline Columbina picui & 7,1 & 4,6 & 8,8 & GRA & IND & Thamnophilus capistratus & 15,0 & 0,4 & 8,8 & INS & SDE \\
\hline Claravis pretiosa & - & - & 1,7 & FRU & SDE & Taraba major & 20,4 & 2,1 & 29,2 & INS & SDE \\
\hline Leptotila verreauxi & 7,1 & 0,8 & 15,8 & FRU & SDE & Dendrocolaptidae & & & & & \\
\hline Psittacidae & & & & & & Dendroplex picus & 2,5 & - & 3,3 & INS & SDE \\
\hline Forpus xanthopterygius & 5,4 & 2,5 & 3,8 & FRU & IND & Furnariidae & & & & & \\
\hline Cuculidae & & & & & & Furnarius figulus & 0,4 & - & - & INS & IND \\
\hline Piaya cayana & 0,8 & - & 0,8 & INS & SDE & Furnarius leucopus & 1,7 & 1,3 & 3,3 & INS & SDE \\
\hline C. melacoryphus & 1,3 & - & 1,3 & INS & SDE & Pseudoseisura cristata & 3,3 & 3,3 & 4,6 & INS & IND \\
\hline
\end{tabular}

Categorias tróficas (CT): AQUA = organismos aquáticos; CAR = carnívoros; DET = detritívoros; FRU = frugívoros; GRA = granívoros; INS = insetívoros; $\mathrm{NEC}=$ nectarívoros; ONI = onívoros $)$. Uso de habitat $(\mathrm{UH}): \mathrm{DEP}=$ Dependentes de ambientes florestais; SDE = Semi-dependentes de ambientes florestais; IND $=$ Independente de ambientes florestais). Espaços com hífen ( - ) significam FO $\%=0$ 
Tabela 1. (continuação) Lista de espécies de aves classificadas por ordem sistemática registradas no Parque Estadual do Pico do Jabre, Paraíba e suas respectivas frequências de ocorrência (FO\%) nos diferentes ambientes, categorias tróficas (CT) e uso de habitat (UH).

\begin{tabular}{|c|c|c|c|c|c|c|c|c|c|c|c|}
\hline \multirow[b]{2}{*}{ Espécie } & \multicolumn{3}{|c|}{ FO\% da Área } & \multirow[b]{2}{*}{ CT } & \multirow[b]{2}{*}{$\mathrm{UH}$} & \multirow[b]{2}{*}{ Espécie } & \multicolumn{3}{|c|}{ FO\% da Área } & \multirow[b]{2}{*}{$\mathrm{CT}$} & \multirow[b]{2}{*}{$\mathrm{UH}$} \\
\hline & Arbórea & $\begin{array}{c}\text { Área } \\
\text { aberta }\end{array}$ & Floresta & & & & Arbórea & $\begin{array}{c}\text { Área } \\
\text { aberta }\end{array}$ & Floresta & & \\
\hline Gyalophylax hellmayri & 0,8 & - & 0,4 & INS & SDE & Polioptilidae & & & & & \\
\hline Synallaxis frontalis & 13,8 & 2,5 & 15,4 & INS & DEP & Polioptila plumbea & 2,5 & - & 1,7 & INS & SDE \\
\hline Synallaxis scutata & 7,1 & 0,4 & 10,8 & INS & SDE & Turdidae & & & & & \\
\hline Synallaxis frontalis & 13,8 & 2,5 & 15,4 & INS & DEP & Turdus rufiventris & 3,8 & 0,8 & 8,8 & ONI & IND \\
\hline Synallaxis scutata & 7,1 & 0,4 & 10,8 & INS & SDE & Turdus leucomelas & 1,3 & - & 0,4 & ONI & SDE \\
\hline Tyrannidae & & & & & & Turdus amaurochalinus & 0,4 & - & 1,3 & ONI & SDE \\
\hline Tolmomyias flaviventris & 7,5 & 0,8 & 7,9 & INS & DEP & Mimidae & & & & & \\
\hline Todirostrum cinereum & 6,3 & 0,4 & 5,0 & INS & SDE & Mimus saturninus & 2,5 & 5,0 & 2,9 & ONI & IND \\
\hline Hemitriccus margaritaceiventer & 10,8 & 0,8 & 8,8 & INS & SDE & Coerebidae & & & & & \\
\hline Hirudinea ferruginea & 3,8 & - & 4,6 & INS & SDE & Coereba flaveola & 0,8 & 0,4 & 0,4 & NEC & SDE \\
\hline Camptostoma obsoletum & 0,4 & - & - & INS & IND & Thraupidae & & & & & \\
\hline Elaenia spectabilis & 4,2 & 0,8 & 5,4 & FRU & DEP & Lanio pileatus & 6,7 & 0,8 & 10,4 & ONI & SDE \\
\hline Elaenia chilensis & & - & 0,4 & ONI & IND & Tangara sayaca & 3,8 & 2,1 & 10,4 & ONI & SDE \\
\hline Elaenia cristata & 0,4 & - & & ONI & IND & Tangara cayana & 4,6 & - & 9,2 & FRU & IND \\
\hline Elaenia chiriquensis & 0,4 & - & - & ONI & IND & Paroaria dominicana & 3,3 & 2,5 & 2,1 & GRA & IND \\
\hline Myiopagis viridicata & 5,0 & 0,8 & 12,9 & FRU & DEP & Conirostrum speciosum & - & - & 0,8 & INS & DEP \\
\hline Phaeomyias murina & 5,4 & 0,4 & 2,9 & ONI & SDE & Emberizidae & & & & & \\
\hline Legatus leucophaius & - & & 0,4 & FRU & DEP & Zonotrichia capensis & 0,4 & - & - & $\overline{\text { GRA }}$ & IND \\
\hline Pitangus sulphuratus & 4,6 & 2,5 & 1,7 & ONI & IND & Volatinia jacarina & 5,0 & 3,3 & 5,4 & GRA & IND \\
\hline Megarynchus pitangua & 1,3 & 0,8 & 2,9 & INS & SDE & Sporophila lineola & 1,7 & 0,0 & 0,8 & GRA & IND \\
\hline Myiozetetes similis & 4,6 & 0,8 & 8,8 & ONI & SDE & Sporophila nigricollis & 17,1 & 4,2 & 20,0 & GRA & IND \\
\hline Tyrannus melancholicus & 22,1 & 6,7 & 24,2 & INS & IND & Sporophila albogularis & 1,7 & 2,1 & 0,4 & GRA & IND \\
\hline Empidonomus varius & 5,4 & 0,4 & 10,0 & INS & SDE & Arremon taciturnus & 7,1 & - & 20,4 & ONI & DEP \\
\hline Fluvicola nengeta & 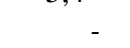 & 0,4 & - & INS & IND & Cardinalidae & & & & & \\
\hline Arundinicola leucocephala & - & 0,8 & - & INS & IND & Cyanoloxia brissonii & 0,8 & - & 0,4 & GRA & DEP \\
\hline Cnemotriccus fuscatus & 1,3 & - & 1,7 & INS & DEP & Parulidae & & & & & \\
\hline Vireonidae & & & & & & Basileuterus flaveolus & 5,4 & - & 9,2 & DEP & DEP \\
\hline Cyclarhis gujanensis & 11,7 & 2,5 & 18,8 & INS & SDE & Icteridae & & & & & \\
\hline Vireo chivi & - & - & 1,3 & INS & DEP & Icterus cayanensis & 0,8 & - & - & ONI & SDE \\
\hline Hylophilus amaurocephalus & 9,2 & - & 15,4 & INS & DEP & Icterus jamacaii & - & 2,9 & 0,8 & ONI & SDE \\
\hline Corvidae & & & & & & Chrysomus ruficapillus & - & 0,4 & - & ONI & IND \\
\hline Cyanocorax cyanopogon & 2,9 & - & 5,8 & ONI & SDE & Molothrus bonariensis & 1,3 & 1,3 & 1,3 & ONI & IND \\
\hline Tachycineta albiventer & - & 0,4 & - & INS & IND & Fringillidae & & & & & \\
\hline Troglodytidae & & & & & & Euphonia chlorotica & 5,4 & 1,7 & 12,9 & FRU & SDE \\
\hline Troglodytes musculus & 5,0 & 0,8 & 8,3 & INS & IND & & & & & & \\
\hline Canthorchilus longirostris & 19,6 & 1,7 & 36,3 & INS & DEP & & & & & & \\
\hline
\end{tabular}

Categorias tróficas $(\mathrm{CT})$ : AQUA = organismos aquáticos; CAR = carnívoros; DET = detritívoros; FRU = frugívoros; GRA = granívoros; INS = insetívoros;

$\mathrm{NEC}=$ nectarívoros; ONI $=$ onívoros). Uso de habitat $(\mathrm{UH})$ : DEP $=$ Dependentes de ambientes florestais; SDE $=$ Semi-dependentes de ambientes florestais;

IND = Independente de ambientes florestais). Espaços com hífen ( - ) significam FO $\%=0$. 


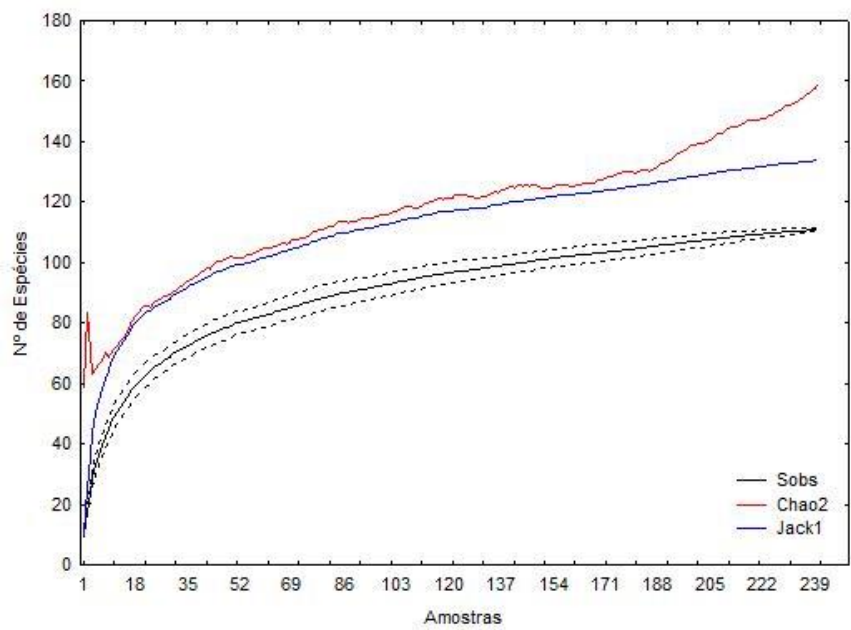

Figura 1. Curva de acumulação de espécies, Chao 2 e Jack 1 das espécies de aves registradas no Parque Estadual do Pico do Jabre, Paraíba.

Quanto ao uso de habitat, 19,8\% das espécies que ocorrem na área se mostraram dependentes de ambientes florestais, $38,7 \%$ semi-dependentes e $41,4 \%$ independentes. Das espécies dependentes de ambientes florestais, quatro delas foram registradas apenas uma vez na área estudada (FO\% = $0,4 \%$; Tabela 1). Com exceção de $P$. perspicilata (Latham, 1790), que apresenta hábitos noturnos, nenhuma dessas espécies apresentam uma difícil detecção pelo método utilizado. Podemos assumir esse fator a uma densidade naturalmente baixa, entretanto, a população de $P$. jacucaca ainda sofre com pressões de caça, além da perda de habitat.

Apesar da área amostrada compreender uma formação florestal de topo de serra, o padrão observado é o esperado para as áreas de Caatinga. As áreas abertas e antropizadas das regiões mais baixas da área de estudo são as principais contribuintes para essa porcentagem da ocorrência de espécies independentes de florestas e a porção florestal, localizada acima dos $700 \mathrm{~m}$ de altitude, que influencia na ocorrência das espécies dependentes de florestas.

Áreas degradadas ou em regeneração apresentam baixa diversidade de nichos, em consequência disto, espécies generalistas, insetívoras não especialistas, semi-dependentes e independentes de ambientes florestais tornam-se os primeiros colonizadores, o que pode justificar a distribuição de espécies com distintos usos de habitat na área de estudo.

No conjunto das espécies registradas, insetívoros foram predominantes $(39,6 \%)$, seguida das espécies onívoras $(24,3 \%)$ e frugívoras (9\%; Figura 2).

Muitos estudos (Willis, 1979; Anjos, 1998; Lyra-Neves et al., 2004; Telino-Júnior et al., 2005; Rodrigues et al., 2007) registraram um padrão semelhante ao aqui observado, no qual espécies pertencentes às categorias tróficas citadas são predominantes em bordas florestais, capoeiras, áreas de mata e/ou em estado de regeneração.

A presença de espécies frugívoras de relativo grande porte, como os Tinamidae, Cracidae e as pombas Claravis pretiosa (Ferrari-Perez, 1886) e L. verreauxi Bonaparte, 1855, podem ser fundamentais para a manutenção de fragmentos florestais devido a sua capacidade de deslocamento em busca de recursos alimentares e, consequentemente, tornando-se potenciais dispersores de sementes (Lyra-Neves et al., 2004). Em áreas impactadas ou que sofrem constante uso pelo homem, pode-se verificar a ocorrência de aves com maior espectro alimentar, como onívoros. Isso ocorre provavelmente pela possibilidade da mudança de dieta em períodos adversos (Willis, 1979).

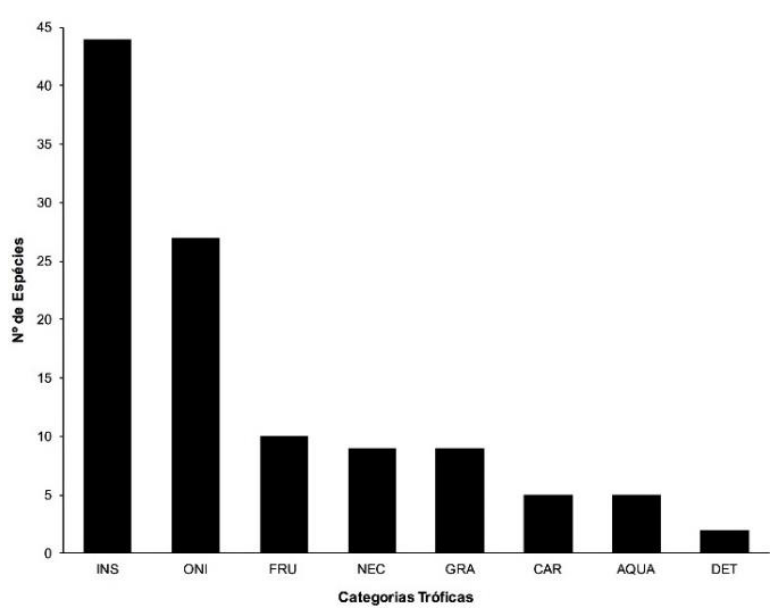

Figura 2. Número de espécies de aves quanto a categoria trófica no Parque Estadual do Pico do Jabre, PB. AQUA: Hábitos aquáticos; CAR: Carnívoras; DET: Detritívas; FRU: Frugívora; GRA: Granívora; INS: Insetívora; NEC: Nectarívora; ONI: Onívora.

A fragmentação e o consequente efeito de borda beneficiam grupos granívoros como os Columbidae de pequeno porte (Anjos, 1998), devido à maior incidência de luz nesses ambientes proporcionar maior produção de frutos e plantas invasoras produtoras de sementes, base alimentar dessas espécies. Desta forma, justifica-se a presença de espécies com baixa dependência de ambientes florestais e de espectro alimentar mais generalistas nas porções inferiores do Pico do Jabre.

$\mathrm{O}$ pequeno número de espécies aquáticas ou ribeirinhas registradas nessa pesquisa se deve pela ausência de corpos d’água no dentro e no entorno do parque.

\section{Conclusão}

A formação florestal do Parque Estadual do Pico do Jabre ainda se mostra bem preservada, fornecendo indícios de uma riqueza potencialmente maior. A presença de espécies dependentes florestais, de frugívoros e/ou com algum grau de ameaça que utilizam o Parque, como as espécies dos gêneros Crypturellus e Penelope e algumas espécies de Tyrannidae, mostram a importância da área florestal para a manutenção da diversidade da comunidade de aves em seu entorno.

Apesar da importância de se realizar o primeiro levantamento da avifauna no parque, tendo em vista que a informação bibliográfica da composição desta biocenose das florestas de altitude do Nordeste é escassa, há necessidades de um levantamento mais aprofundado e com amostragens sazonais, visando o suporte para pesquisas e planos de manejo futuros. 


\section{Agradecimentos}

As atividades de campo deste trabalho foram incluídas nas coletas de dados do projeto Relações Biogeográficas Entre a Avifauna de Florestas de Altitude no Nordeste do Brasil", financiado pelo Conselho Nacional de Pesquisa (CNPQ) Edital MCT/CNPq No 014/2010 - Universal, Processo $\mathrm{N}^{\circ}$ 478907/2010-9.

\section{Referências}

Ab'Saber, A.N. 1970. Províncias geológicas e domínios morfoclimáticos no Brasil. Geomorfologia 20: 1-26.

Ab'Saber, A.N. 1977. Espaços ocupados pela expansão dos climas secos na América do Sul por ocasião dos períodos glaciais Quaternários. Revista do Instituto de Geografia - Série Paleoclimas 3:1-18.

Agra, M. F.; Barbosa, M.R. de V.; Stevens, W.D. 2004. Levantamento Florístico Preliminar do pico do Jabre, Paraíba, Brasil. In: Porto, K.C.; Cabral, J.P.; Tabarelli, M. (Eds.). Brejos de altitude de Pernambuco e Paraíba: história natural, ecologia e conservação. Brasília-DF: Ministério do meio Ambiente. p.123-137.

Andrade-Lima, D. 1982. Present-day Forest refuges in northeastern Brazil. In: Prance (ed.). Biological diversification in the tropics. Columbia Univ. Press, New York. p. 123-135.

Anjos, L., 1998. Consequências biológicas da fragmentação no norte do Paraná. IPEF, Piracicaba 12, (32): 87-94.

Araujo, H.F.P. Vieira-Filho, A.; Cavalcanti, T.A.; Barbosa, M.R.V. 2012. As aves e os ambientes em que elas ocorrem em uma reserva particular no cariri paraibano, nordeste do Brasil. Revista Brasileira de Ornitologia (Online), v. 20, p. 365-377.

Bibby, C., Jones, M., Marsden, S. 1998. Expedition Field Techniques: Bird Surveys. Expedition Adisory Centre, London.

Colwell, R. K. 2005. User's guide to EstimateS7.5 statistical. Estimation of species richness and shared species from samples. Version 7.5. Copyright $2005,22 \mathrm{p}$.

IBGE - Instituto Brasileiro de Geografia e Estatística (Rio deJaneiro, RJ). Atlas do Brasil: região Nordeste. Rio de janeiro, 1985

Lyra-neves, R.M., S.M. Azevedo júnior, W. Telino-junior, W., M.E.L Larrazábal. 2004. Comunidade de aves da Reserva Estadual de Gurjaú, Pernambuco, Brasil. Revista Brasileira de Zoologia, 21(3): 581-592.

Mackinnon, J.R. e Philipps, K. 1993. A field guide to the birds of Borneo, Sumatra, Java, and Bali, the Greater Sunda Islands. Oxford University Press.

Oliveira-Filho A.T.; Fontes, M.A.L. 2000. Patterns of floristic differentiation among Atlantic Forest in Southeastern Brazil and the influence of climate. Biotropica, v.32, n.4b, p.793-810.

Rodal M.J.N., M.R.V Barbosa e W.W. Thomas. 2008. Do the seasonal forests in northeastern Brazil represent a single floristic unit? Brazilian Journal Biology 68(3): 467-475.

Rodrigues R., Araujo H., Lyra-neves R., Telino-Júnior W. e Botelho M. 2007. Caracterização da Avifauna na Área de Proteção Ambiental de Guadalupe, Pernambuco. Ornithologia, 2, (1): 47-61.

Santos A.M.M. Cavalcanti D.R. Silva J.M.C. e Tabarelli M. 2007. Biogeographical relationships among tropical forests in north-eastern Brazil. Journal of Biogeography 34: 437-466.

Santos, M.P.D., Cerqueira, P.V. e Soares, L. M. S.2010. Avifauna em seis localidades no Centro-Sul do Estado do Maranhão, Brasil. Ornithologia $4(1): 49-65$

Silva, J. M. C., Souza, M. A., Bieber, A. G. D. e Carlos, C. J. 2003. Aves da Caatinga: status, uso do habitat e sensitividade. In: Leal, I. R., Tabarelli, M. e Silva, J. M. C. 2003. Ecologia e Conservação da Caatinga. Recife: Ed Universitária da UFPE. p. 237-273

Silveira, L. F. e F.C. Straube. F. 2008. Livro Vermelho da Fauna Brasileira Ameaçada de Extinção. In: Machado, A.B.M., G.M. Drummond e A.P. Paglia (eds.). Aves ameaçadas de extinção no Brasil. Ministério do Meio Ambiente/Fundação Biodiversitas, Brasília, 2008. p.379-666

Tabarelli M. e Santos, A.M.M. 2004. Uma breve descrição sobre a história natural dos brejos nordestinos. In: Porto K., J. Cabral e M. Tabarelli. Brejos de altitude: história natural, ecologia e conservação. Brasília,
Ministério do Meio Ambiente p. 17-24

Telino-Junior, W., M.M. Dias, S.M. Azevedo Júnior, R.M. Lyra-Neves, e M.E.L. Larrazábal. 2005. Estrutura trófica da reserva estadual do Gurjaú, Zona da Mata Sul, Pernambuco, Brasil. Revista Brasileira de Zoologia, Curitiba 22 (4): 962-973,

Whitman, A.A., Hagan III, J.M. e Brokaw, N.V.L. 1997. A comparasion of two bird survey techniques used in a subtropical forest. The Condor, 99: 955-965.

Willis, E.O., 1979. The composition of avian communities in remanescent woodlots in southern Brazil. Papéis Avulsos de Zoologia, São Paulo 33: $1-25$. 